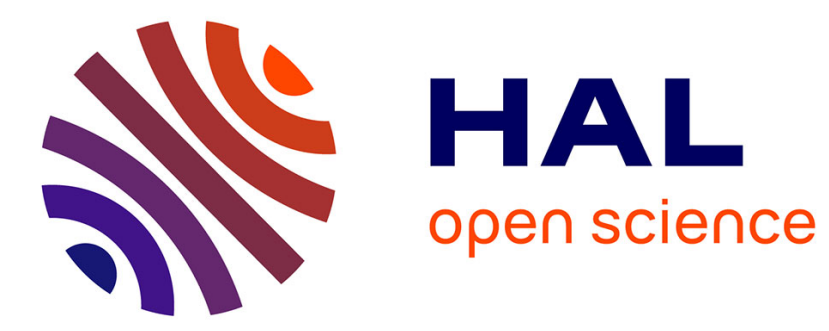

\title{
Permeability models affecting nonlinear stability in the asymptotic suction boundary layer: the Forchheimer versus the Darcy model
}

Håkan Wedin, Stefania Cherubini

\section{- To cite this version:}

Håkan Wedin, Stefania Cherubini. Permeability models affecting nonlinear stability in the asymptotic suction boundary layer: the Forchheimer versus the Darcy model. Fluid Dynamics Research, 2016, 48 (6), pp.1-17. 10.1088/0169-5983/48/6/061411 . hal-02569805

\section{HAL Id: hal-02569805 \\ https://hal.science/hal-02569805}

Submitted on 11 May 2020

HAL is a multi-disciplinary open access archive for the deposit and dissemination of scientific research documents, whether they are published or not. The documents may come from teaching and research institutions in France or abroad, or from public or private research centers.
L'archive ouverte pluridisciplinaire HAL, est destinée au dépôt et à la diffusion de documents scientifiques de niveau recherche, publiés ou non, émanant des établissements d'enseignement et de recherche français ou étrangers, des laboratoires publics ou privés. 


\title{
Permeability models affecting nonlinear stability in the asymptotic suction boundary layer: the Forchheimer versus the Darcy model
}

\author{
Håkan Wedin ${ }^{1}$ and Stefania Cherubini ${ }^{2,3}$ \\ ${ }^{1}$ Department of Civil, Chemical and Environmental Engineering, University of \\ Genova, Via Montallegro 1, I-16145 Genova, Italy \\ ${ }^{2}$ DynFluid, Arts et Métiers ParisTech, 151 Bd. de l'Hôpital, F-75013 Paris, France ${ }^{3}$ \\ DMMM, Politecnico di Bari, Via Re David 200, I-70125, Bari, Italy \\ E-mail: hakanwedin@hotmail.com \\ Communicated by L Tuckerman
}

\begin{abstract}
The asymptotic suction boundary layer (ASBL) is used for studying two permeability models, namely the Darcy and the Forchheimer model, the latter being more physically correct according to the literature. The term that defines the two apart is a function of the non-Darcian wall permeability $\hat{K}_{2}$ and of the wall suction $\hat{V}_{0}$, whereas the Darcian wall permeability $\hat{K}_{1}$ is common to the two models. The underlying interest of the study lies in the field of transition to turbulence where focus is put on two-dimensional nonlinear traveling waves (TWs) and their three-dimensional linear stability. Following a previous study by Wedin et al (2015 Phys. Rev. E 92 013022), where only the Darcy model was considered, the present work aims at comparing the two models, assessing where in the parameter space they cease to produce the same results. For low values of $\hat{K}_{1}$ both models produce almost identical TW solutions. However, when both increasing the suction $\hat{V}_{0}$ to sufficiently high amplitudes (i.e. lowering the Reynolds number $R e$, based on the displacement thickness) and using large values of the wall porosity, differences are observed. In terms of the non-dimensional Darcian wall permeability parameter, $a$, strong differ-

ences in the overall shape of the bifurcation curves are observed for $a \gtrsim 0.70$, with the emergence of a new family of solutions at Re lower than 100 . For these large values of $a$, a Forchheimer number $F o_{\max } \gtrsim 0.5$ is found, where $F o$ expresses the ratio between the kinetic and viscous forces acting on the porous wall. Moreover, the minimum Reynolds number, $R e_{g}$, for which the Navier-
\end{abstract}


Stokes equations allow for nonlinear solutions, decreases for increasing values of $a$. Fixing the streamwise wavenumber to $\alpha=0.154$, as used in the study by Wedin $e t a l$ referenced above, we find that $R e_{g}$ is lowered from $R e \approx 3000$ for zero permeability, to below 50 for $a=0.80$ for both permeability models. Finally, the stability of the TW solutions is assessed using a three-dimensional linearized direct numerical simulation (DNS). Low-frequency unstable modes are found for both permeability models; however, the Darcy model is found to overpredict the growth rate, and underpredict the streamwise extension of the most unstable mode. These results indicate that a careful choice of the underlying permeability model is crucial for accurately studying the transition to turbulence of boundary-layer flows over porous walls.

Keywords: transition to turbulence, coherent structures, direct numerical simulation, instabilities, dynamical system approaches

(Some figures may appear in colour only in the online journal)

\section{Introduction}

The first studies on flows through circular pipes were independently carried out by Hagen (1839) and Poiseuille (1846). Based on the works of Hagen and Poiseuille, the pioneering study on the transition to turbulence goes back more than 130 years to the well-known laboratory experiments of Osborne Reynolds (1883) on incompressible pipe flow. Later Rayleigh (1892) and Reynolds (1895) published the two first theoretical studies on hydrodynamical stability; these were followed by the seminal papers of Tollmien (1929) and

Schlichting (1933). Before the 1940s turbulent flows were perceived as a chaotic and random motion, however the experiments of Emmons and Bryson (Emmons (1951), Emmons and Bryson (1952)) suggested that they are characterized by organized formations such as spots; Kline et al (1967) and Acarlar and Smith (1987a, 1987b) observed recurrent structures that have the shape of vortices and streaks, which today are usually referred to as coherent flow structures. On the theoretical side, Nagata (1990) presented for the first time numerical exact invariant solutions to the Navier-Stokes equations, usually labeled as global or periodic as opposed to localized solutions. Subsequently, a large body of literature has focused on global flow states (Waleffe (1998, 2001, 2003), Wedin et al (2014, 2009), Uhlmann et al (2010), Wedin and Kerswell (2004), Faisst and Eckhardt (2003), Pringle et al (2009), Itano and Generalis (2009), Deguchi and Hall (2014)), local invariant solutions such as equilibrium states (Brand and Gibson (2014), Gibson and Brand (2014)) as well as periodic orbits (Kawahara and Kida (2001), Chandler and Kerswell (2013), Chantry et al (2014), Lucas and Kerswell (2015)) based on the periodic orbit theory (Cvitanović et al (2012), Cvitanović (2013)). Each type of nonlinear solution mentioned above is capable of capturing certain aspects of turbulent flows, providing important indications on how turbulence is sustained, and how this self-sustaining cycle can be unfolded in order to control this chaotic motion.

A good example of how to control turbulence is wall suction applied on boundary-layer flows, with the purpose of damping perturbations possibly responsible for the turbulent transition. Applying a homogeneous suction on a Blasius boundary layer (BBL) flow, one obtains the asymptotic suction boundary layer (ASBL) flow, which is a much more stable laminar solution of the Navier-Stokes equations. A demonstration of this is its very high linear stability limit in the Reynolds number based on the displacement thickness, 


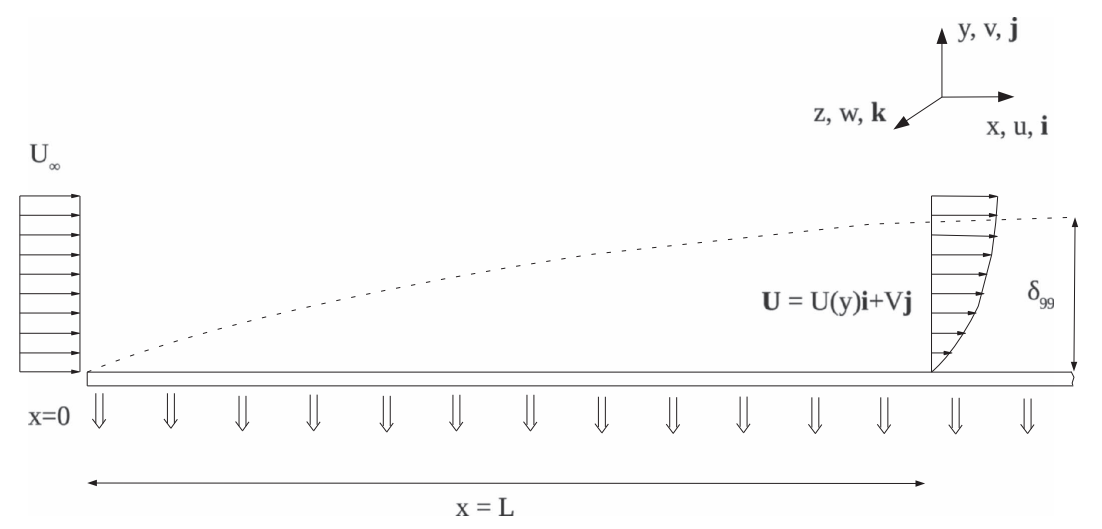

Figure 1. A sketch of a boundary-layer flow with homogeneous suction at the wall. The incoming uniform flow at the left has streamwise speed $\hat{U}_{\infty}$. After a certain length in the downstream direction the boundary-layer flow reaches asymptotically the state given in equation $(1 a)$. The $\delta_{99}$ refers to the thickness of the boundary layer.

$R e_{c} \approx 54400$ (Hocking (1975), Fransson (2003)), whereas the laminar BBL flow with no suction is unstable to small amplitude perturbations from $R e_{c}=520$ (Schmid and Henningson (2001)). Concerning nonlinear stability, Cherubini et al (2015) found that, neglecting the influence of wall permeability on the perturbations, the ASBL minimal energy thresholds for transition are one order of magnitude larger than those of the BBL. However, for sufficiently large wall permeability $\hat{K}_{1}$ of the ASBL, and comparing it with the BBL, Wedin et al (2015) have confirmed the stabilizing effect of wall suction on perturbations of low amplitude, whereas finite-amplitude disturbances have been found to reach further down in $R e$ with respect to the Blasius flow. The linear study of Pluvinage et al (2015) confirms the findings outlined in Wedin et al (2015), observing that even small values (above a certain threshold) of the permeability greatly alter the stability characteristics of the ASBL. In this study we use a selection of theoretical techniques used for studying the transition to turbulence, to compute nonlinear traveling waves (TWs) solutions for the ASBL. In particular, focus is here put on the effect on the TWs and the linear stability of two different models of wall permeability: the Darcy permeability model recently considered in Wedin et al (2015), Pluvinage et al (2015) and a more realistic model, the Forchheimer description of the wall porosity (see Innocentini et al (2005)). The aim is to point out discrepancies between the Darcy and the Forchheimer model in the analysis of the nonlinear stability of flows over porous walls as either the permeability $\hat{K}_{1}$ or the wall suction $\hat{V}_{0}$ is altered. The paper is organized as follows. In section 2 the flow configuration, equations and formulas are presented. Section 3 presents two-dimensional Tollmien-Schlicting (TS) waves followed by a three-dimensional stability analysis. Lastly, conclusions can be found in section 4 .

\section{Definitions}

An incompressible flow $\hat{\boldsymbol{u}}=(\hat{u}, \hat{v}, \hat{w})$ of constant density $\hat{\rho}$, dynamic viscosity $\hat{\mu}$, and kinematic viscosity $\hat{\nu}=\hat{\mu} / \hat{\rho}$ over a porous plate is studied. The streamwise, wall-normal, and spanwise directions are denoted as $\hat{x}, \hat{y}$, and $\hat{z}$. The lower and upper boundaries of the computational domain are situated respectively at $\hat{y}=0$ and $\hat{y}=\hat{y}_{\infty}$, where the constant flow speed in the streamwise direction equals the freestream velocity $\hat{U}_{\infty}$. Between these extreme 
points a boundary layer exists, as sketched in figure 1 . When a uniform wall-normal suction velocity, $\hat{V}_{0}$, is imposed through the porous plate, a parallel flow called the ASBL is observed. In a zero-disturbance environment the flow remains two-dimensional and laminar, as given by the steady solution $\hat{\boldsymbol{U}}=(\hat{U}, \hat{V}, 0)$, where:

$$
\begin{aligned}
& \hat{U}(\hat{y})=\hat{U}_{\infty}\left[1-e^{-\hat{V}_{0} \hat{y} / \hat{\nu}}\right], \\
& \hat{V}=-\hat{V}_{0}=\text { constant, } \quad \text { with } \hat{V}_{0}>0 .
\end{aligned}
$$

On top of this steady laminar flow, finite-amplitude perturbations might evolve in the form of TWs, which are relative equilibria in a frame of reference moving with a fixed phase velocity (Wedin and Kerswell (2004)). In this study we focus on the two-dimensional flow case (hence the spanwise velocity component $\hat{w}$ is set to zero), computing nonlinear TS TWs over a porous flat plate of thickness $\hat{h}$ using two different permeability laws governing the flow through the porous medium placed at $\hat{y}=0$. The nonlinear TWs sought for are solutions of the incompressible Navier-Stokes equations:

$$
\begin{aligned}
& \frac{\partial \hat{\boldsymbol{u}}}{\partial \hat{t}}+(\hat{\boldsymbol{u}} \cdot \hat{\boldsymbol{\nabla}}) \hat{\boldsymbol{u}}=-\frac{1}{\hat{\rho}} \hat{\boldsymbol{\nabla}} \hat{p}+\frac{\hat{\mu}}{\hat{\rho}} \hat{\boldsymbol{\nabla}}^{2} \hat{\boldsymbol{u}}, \\
& \hat{\boldsymbol{\nabla}} \cdot \hat{\boldsymbol{u}}=0 .
\end{aligned}
$$

To find these solutions bifurcating from the laminar flow $\hat{\boldsymbol{U}}(\hat{y})$ we impose a perturbation $\hat{\boldsymbol{u}}^{\prime}=\left(\hat{u}^{\prime}, \hat{v}^{\prime}, 0\right)$ having the form of a TW solution $\hat{\boldsymbol{u}}_{1}{ }_{1}$, where index 1 stands for the primary bifurcation computed in this study and $\epsilon$ is an unknown amplitude to be computed. To represent the TW as a sum of monochromatic waves, a fundamental TS wave is considered, having the eigenvalue $\hat{c}=\hat{c}_{r}+i \hat{c}_{\mathrm{i}}$ ( $\mathrm{i}$ being the imaginary constant) and fundamental streamwise wavenumber $\hat{\alpha}$. This monochromatic wave, characterized by only one temporal frequency, grows/decays and oscillates in time with the growth rate $\hat{\alpha} \hat{c}_{\text {i }}$ and frequency $\hat{\alpha} \hat{c}_{r}$. The TW $\boldsymbol{\epsilon}_{{ }^{\prime}}{ }_{1}$ is thus represented as a sum of TS waves with streamwise and temporal wavenumbers being multiples of the fundamental ones, using a Fourier series in $\hat{x}$ to enforce periodic conditions and a Chebyshev series in the wall-normal direction, as follows:

$$
\begin{aligned}
& \hat{\boldsymbol{u}}_{1}^{\prime}=\sum_{b} \hat{\boldsymbol{u}}^{(b)}(\hat{y}) \exp [i b \hat{\alpha}(\hat{x}-\hat{c} \hat{t})], \\
& =\sum_{b} \sum_{j} \hat{\boldsymbol{v}}_{b j} T_{j}(\hat{y}) \exp [i b \hat{\alpha}(\hat{x}-\hat{c} \hat{t})],
\end{aligned}
$$

for $b=-N X, \ldots,+N X$. Since $\hat{\mathrm{a} t}=\hat{y}_{\infty}$ the instantaneous streamwise velocity must equal the freestream velocity $\hat{U}_{\infty}$, any perturbation should decay as $\hat{y} \infty \rightarrow$; thus, the zeroth Fourier mode $\hat{v}^{(b=0)}$ must be set to zero. At the plate $\hat{y}=0$ we impose a no-slip condition on $\hat{u}^{\prime}$; the majority of studies also assume zero velocity on the $\hat{v}^{\prime}$ component (Milinazzo and Saffman (1985), Kreilos et al (2013), Khapko et al (2013), Gustavsson (2000), Fransson (2003), Fransson and Corbett (2003), Levin et al (2005), Davidsson (2007)). This is a good approximation as long as the permeability of the porous medium is sufficiently low, as shown by Wedin et al (2015). However, when the wall porosity is sufficiently high, one should take into account the presence of a non-zero $\hat{v}^{\prime}$ component of the perturbation at the wall, using a porosity model such as the Darcy or the Forchheimer permeability model (Innocentini et al (2005)). For the vertical laminar viscous flow through the porous medium, the relation between the pressure and the wall suction is linear according to Darcy's law, as shown in equation $(4 a)$. However, for an inertial flow where the wall suction is further increased or alternatively is at a certain threshold value of the permeability $\hat{K}_{1}$ (also called the Darcian 


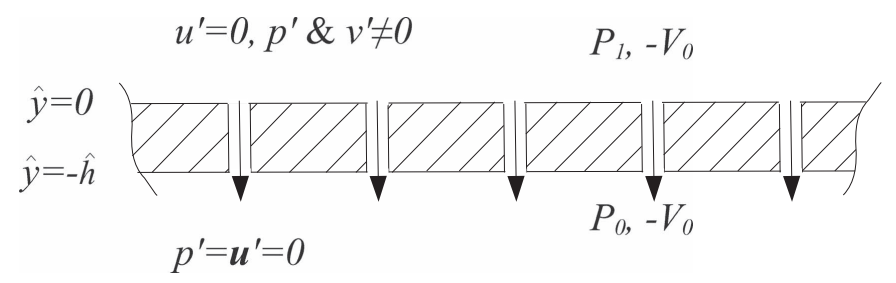

Figure 2. A sketch of the porous wall of dimensional thickness $\hat{h}$ along with the imposed conditions at the wall. Base flow variables are indicated by capital letters, whereas primes indicate perturbations.

permeability $\left[\mathrm{m}^{2}\right]$ ), the Darcy model may not give the correct dependence between the pressure and the wall velocity. This is the case where the Forchheimer model might be preferable (Reynolds (1900), Forchheimer (1901), Innocentini et al (1999, 2005, 2012)), providing a nonlinear relationship between the pressure gradient $\mathrm{d} \hat{P} / \mathrm{d} \hat{y}$ and the average suction velocity $\hat{V}$ according to the formula in equation (4b) outlined below:

$$
\begin{array}{ll}
-\frac{\mathrm{d} \hat{P}}{\mathrm{~d} \hat{y}}=\frac{\hat{\mu}}{\hat{K}_{1}} \hat{V}, & (\text { Darcy) }, \\
-\frac{\mathrm{d} \hat{P}}{\mathrm{~d} \hat{y}}=\frac{\hat{\mu}}{\hat{K}_{1}} \hat{V}+\frac{\hat{\rho}}{\hat{K}_{2}} \hat{V}^{2} & \text { (Forchheimer). }
\end{array}
$$

In order to set a value for the non-Darcian permeability $\hat{K}_{2}([m])$, also called the inertial permeability, we use the permeability data given in Innocentini et al (2005) corresponding to an experimentally-determined relation between $\hat{K}_{1}$ and $\hat{K}_{2}$. The formula given below originates from a large quantity of permeability data for a considerable amount of porous materials:

$$
\frac{\hat{K}_{2}}{[m]}=\exp \left\{-\frac{1.71588}{\left(\frac{\hat{K}_{1}}{\left[m^{2}\right]}\right)^{0.08093}}\right\} .
$$

One can notice that the quantities in equation (5) have different units. However, the unit conversion is implicit and the formula is purely empirical where constants 1.71588 and 0.08093 are based on a data fitting in which $\hat{K}_{1}$ must be given in $\left[\mathrm{m}^{2}\right]$ in order for $\hat{K}_{2}$ to result in $[m]$. To render the exponential term dimensionless, see equation (5), we divide $\hat{K}_{1}$ by its dimension, i.e. $\left[\mathrm{m}^{2}\right]$. In order to refer our results to an experimental case, we use the parameter setting of Fransson and Alfredsson (2003) where $\hat{U}_{\infty}=5 \mathrm{~ms}^{-1}, \hat{\nu}=1.5 \times 10^{-5} \mathrm{~m}^{2} \mathrm{~s}^{-1}$ and $\hat{h}=3.2 \mathrm{~mm}$, where $\hat{h}$ is the thickness of the plate.

The governing equations are non-dimensionalized (thus removing the caret symbols) using the freestream speed $\hat{U}_{\infty}$ and the displacement thickness $\hat{\delta}_{*}$, whereas the pressure is rendered non-dimensional using $\hat{\rho} \hat{U}_{\infty}^{2}$. This leads to the definition of the Reynolds number $R e=\hat{U}_{\infty} \hat{\delta}_{*} / \hat{\nu}$. Using the definition of the displacement thickness $\hat{\delta}_{*}=\hat{\nu} / \hat{V}_{0}$ we find that $R e=\hat{U}_{\infty} / \hat{V}_{0}$. The unperturbed suction velocity in its non-dimensional form is thus $-1 / R e$. Imposing a perturbation $\boldsymbol{u}^{\prime}$ on the laminar state, the instantaneous two-dimensional velocity and pressure on the plate surface at $y=0$ are given by $\left(0, V_{0}+v^{\prime}, P_{1}+p^{\prime}\right)$. The dimensional thickness of the plate is denoted by $\hat{h}$ and is shown in figure 2 . At the lower surface of the plate $\hat{y}=-\hat{h}$ the flow is unperturbed, i.e $\left(0-V_{0}, P_{0}\right)$. The Darcy's law shown in 
equation $(4 a)$ in its dimensionless form provides a linear dependence between $v^{\prime}$ and $p^{\prime}$ for the disturbance at $y=0$ :

$$
v^{\prime}=-a p^{\prime}, \quad \text { with } \quad a=\frac{\hat{U}_{\infty} \hat{K}_{1}}{\hat{\nu} \hat{h}}
$$

where $a$ is the dimensionless permeability parameter. Notice that, for a given fluid with fixed freestream velocity, $a$ can be increased by either using a more permeable or a thinner wall. However, in this work we fix the plate thickness $\hat{h}$ to the value experimentally used by Fransson and Alfredsson (2003), implying that a change in $a$ is directly linked to a modification of the wall permeability $\hat{K}_{1}$. In the same way, accounting for inertial effects, the corresponding relationship using the Forchheimer description is:

$$
v^{\prime}=-a p^{\prime}\left(1+\frac{\hat{U}_{\infty}}{\hat{\nu}} \frac{\hat{K}_{1}}{\hat{K}_{2}}\left[v^{\prime}-\frac{2}{R e}\right]\right)^{-1},
$$

which is nonlinear in $v^{\prime}$ when rearranging equation (7) to solve for $p^{\prime}$. Figure 2 shows the flow variables at and below the plate as well as the imposed boundary conditions at $\hat{y}=0$. To infer the Darcy and the Forchheimer wall condition for $v^{\prime}$ one can take the $x$ - derivative of the streamwise momentum equation, replacing $u^{\prime}$ using the continuity equation and the nondimensional relation between $v^{\prime}$ and $p^{\prime}$, as given above. To evaluate the deviation between the Forchheimer and the Darcy model, we focus on the second term on the right-hand side of equation $(4 b)$. Rearranging this term one finds:

$$
-\frac{\mathrm{d} \hat{P}}{\mathrm{~d} \hat{y}}=\frac{\hat{\mu}}{\hat{K}_{1}} \hat{V}(1+F o), \quad F o=\frac{\hat{\rho}}{\hat{\mu}} \frac{\hat{K}_{1}}{\hat{K}_{2}} \hat{V},
$$

where Fo is the Forchheimer number according to Ruth and Ma (1992), and corresponds to the relationship between kinetic and viscous forces adding up to the pressure gradient. To define a Forchheimer number for the non-dimensional perturbed case, the maximum absolute value of the second term in equation (7) is computed:

$$
F o_{\max }=\max _{\forall X} \mid \frac{\hat{U}_{\infty}}{\hat{\nu}} \frac{\hat{K}_{1}}{\hat{K}_{2}}\left[v^{\prime}-\frac{2}{R e} \mid,\right.
$$

where $X$ corresponds to the time-independent Galilean frame $X=x-c_{r} t$ and the domain of $v^{\prime}=v^{\prime}(X, y=0)$ is $0 \leqslant X \leqslant 2 \pi / \alpha$. The $F o_{\max }$ represents a measure of the difference between the two permeability models, bringing together the impact from all parameters $\alpha, a$ and $R e$ into one single number. Using equation (7), i.e $p^{\prime}=-\left(v^{\prime} / a\right)\left(1+F o_{\max }\right)$, we can determine the specific contribution of the viscous and the inertial effects on the total pressure perturbation $p^{\prime}=p_{\text {viscous }}^{\prime}+p_{\text {inertial }}^{\prime}$, i.e $p^{\prime}=p_{\text {viscous }}^{\prime}\left(1+F o_{\max }\right)$, where $p_{\text {viscous }}^{\prime}$ is given by the Darcy's model in equation (6). The Forchheimer equation can be manipulated to show that:

$$
p_{\mathrm{viscous}}^{\prime}=\frac{1}{1+F o_{\max }} p^{\prime},
$$

implying that inertial effects are negligible for $F o_{\max } \ll 1$ since $p_{\mathrm{viscous}}^{\prime} \approx p^{\prime}$ and the two permeability models should yield the same results. In the same manner one can show that viscous effects are of minor importance for $F o_{\max } \gg 1$ through:

$$
p_{\text {inertial }}^{\prime}=\frac{F o_{\max }}{1+F o_{\max }} p^{\prime}
$$


since $p_{\text {inertial }}^{\prime} \approx p^{\prime}$. Thus, when $F o_{\max } \gg 1$ the Forchheimer equation should be used to correctly model the problem.

Once the boundary conditions are correctly imposed using the Darcy or the Forchheimer model, we need to solve a nonlinear system of perturbed equations which can be recast in the form:

$$
\boldsymbol{F}\left(\hat{\boldsymbol{v}}_{b j}, c_{r}, \epsilon ; \boldsymbol{\Omega}\right)=\mathbf{0},
$$

where $\hat{\boldsymbol{v}}_{b j}$ are the unknown Fourier coefficients defined in equation (3) and the vector $\boldsymbol{\Omega}=(\alpha, a, R e)$ contains the independent parameters of the problem. To determine the wave speed $c_{r}$ and the amplitude $\epsilon$, the phase of the solution is fixed by imposing the following normalization condition on the first complex Fourier mode:

$$
v^{(b=1)}(y=3)=1,
$$

which is thus set to be purely real, and TS waves with $\epsilon<0$ are selected. Concerning the other independent parameters of the system, one can notice that a change in $R e$ directly implies a modification of the wall suction $\hat{V}_{0}$, whereas when the wall porosity $\hat{K}_{1}$ or the plate thickness $\hat{h}$ changes the parameter $a$ is affected. Thus, for a given fluid with a fixed freestream velocity, a modification of the parameter $a$ does not have any influence on the base flow velocity profile, but only on the dynamics of the perturbation; whereas a change in Re directly affects the shape of the base flow itself. Moreover, looking at equation (7) one can notice that the inertial term of the Forchheimer description of the porous media depends on both $a$ and

$R e$; thus, at some threshold value of either $a$ (i.e $\hat{K}_{1}$ ) or $R e$ (i.e the suction $\hat{V}_{0}$ ) a deviation from the Darcy model might be expected.

For a given set of independent parameters the nonlinear system (12) is solved using a Fortran subroutine written by Rheinboldt and Burkardt (1983a, 1983b) based on a Newton-

Raphson technique. Once the first nonlinear solution is located the subsequent solutions can be mapped out in the parameter space using the above-mentioned subroutine. When tracing out the solutions one of the parameters is varied, for example $\alpha$, and the others are kept constant. The mentioned code originates from a Fortran program used for searching for TS waves in the BBL. To validate the initial version of the code, we have reproduced the nonlinear twodimensional results contained in Milinazzo and Saffman (1985). Then the code has been adapted to the case of the ASBL flow by adding terms containing the laminar suction velocity. To validate the adapted version of the code, the linear stability of the laminar ASBL has been computed, reproducing the critical Reynolds number $R e_{c} \approx 54400$ found by Hocking (1975) and Fransson (2003) imposing a no-slip condition also on the wall-normal component of the velocity. Finally, for the present study, the code, taking into account the Forchheimer condition at the wall, was validated by matching the results in Wedin et al (2015) for low-plate permeabilities $a$. The nonlinear TS waves computed using this procedure should not be considered turbulent, mainly because they do not possess a spanwise extent, as opposed to the well-known mean spanwise spacing $z^{+}=100$ between low-speed streaks (based on the friction velocity) obtained from experiments on turbulence (Lee et al (1974)). Nevertheless, the TS waves may form part of flow states sitting in the pre-transitional domain or which are involved in the initial phase of the turbulence transition. Indeed, being prone to three-dimensional instability, as will be discussed below, these relative equilibria can even-tually lead the flow to turbulence.

Thus, once the nonlinear TS waves are computed using the two different models, their linear stability has been analyzed by using linearized direct numerical simulations (DNSs). Since the most unstable mode of linear TS waves is usually three-dimensional, we have extended the DNSs to a three-dimensional framework. We therefore chose a computational 
box with dimensions $L_{x} \times L_{y} \times L_{z}=4 \pi / \alpha \times y_{\infty} \times 2 \pi$. On this computational domain, the Navier-Stokes equations, written in a perturbative formulation, are discretized and solved using a finite-difference fractional-step method with second-order accuracy in space and time (Verzicco and Orlandi (1996)). The number of grid points in each direction has been chosen after careful validation. In particular, the grid points have been doubled in each direction until a variation of less than $1 \%$ on the growth rate of the most unstable mode is found. For the reference domain $L_{x} \times L_{y} \times L_{z}=4 \pi / 0.2 \times 70 \times 2 \pi$, a grid with the dimensions $200 \times 180 \times 32$ has been chosen. Moreover, the code has been further validated by matching the results presented in Wedin et al (2015) for low-plate permeabilities $a$, whereas for high permeabilities we have verified that, when initialized with the nonlinear TS waves, the perturbations remain close to this relative equilibria for a long enough time, before departing along the most unstable eigendirection. Concerning the boundary conditions, at the wall either the non-dimensional Darcy law for the wall-normal perturbations given in equation (6) is imposed, or otherwise the Forchheimer model in its linearized form is, i.e.:

$$
v^{\prime}=-a p^{\prime}\left(1-2 \frac{\hat{V}_{0}}{\hat{\nu}} \frac{\hat{K}_{1}}{\hat{K}_{2}}\right)^{-1}
$$

whereas a zero-perturbation condition is used for the streamwise and spanwise velocities. At the upper boundary, all perturbations are set to zero, whereas in the spanwise and streamwise direction periodicity is imposed for the three velocity components. For both permeability models, the simulations have been initialized by superposing to the base flow the TW solution for the streamwise and wall-normal velocity components; the perturbation of this relative fixed point has been initialized by a random zero-mean noise of amplitude $10^{-8}$ for the three velocity components, multiplied by an envelope function centered at $y_{c}=3$, namely $f(y)=y^{2}\left(y_{\infty}-y\right)^{2} \exp \left(-\sigma\left(y-y_{c}\right)^{2}\right)$, with $\sigma=0.1$. The linear time evolution of the perturbations has then been studied, by neglecting the perturbative nonlinear terms in the Navier-Stokes equations. To provide a measure of the energy $E$ of the wavy $x$-dependent part of the perturbation in the more general three-dimensional case the formula outlined below is used:

$$
E=\frac{1}{2} \int_{0}^{2 \pi / \alpha} \int_{0}^{2 \pi / \beta} \int_{0}^{y_{\infty}}\left[u^{\prime 2}+v^{\prime 2}+w^{\prime 2}\right] \mathrm{d} X \mathrm{~d} y \mathrm{~d} z .
$$

where $w^{\prime}=0$ for two-dimensional TS waves, $X$ is the previously-defined time-independent Galilean frame and $z$ and $\beta$ are the spanwise coordinate and spanwise wavenumber respectively.

\section{Results}

\subsection{Nonlinear TS waves}

To gain insight on the limits of the Darcy model, this particular description of the wall permeability is set against the more accurate Forchheimer model. We have chosen to initially restrict the analysis to a two-dimensional case to reduce the size of the parameter space with respect to a three-dimensional case. To search for nonlinear solutions we first solve the linearized governing equations looking for a bifurcation point in the parameter space where the imaginary part of the eigenvalue $c$ is zero $\left(c_{\mathrm{i}}=0\right)$. Using this solution as an initial guess we search for a wave of small but finite amplitude with wave speed $c_{r}$. To start the parameter study in $\alpha$ and $\operatorname{Re}$ we select $\alpha=0.154$ and $a=0$ as initial values, as per one of the cases 

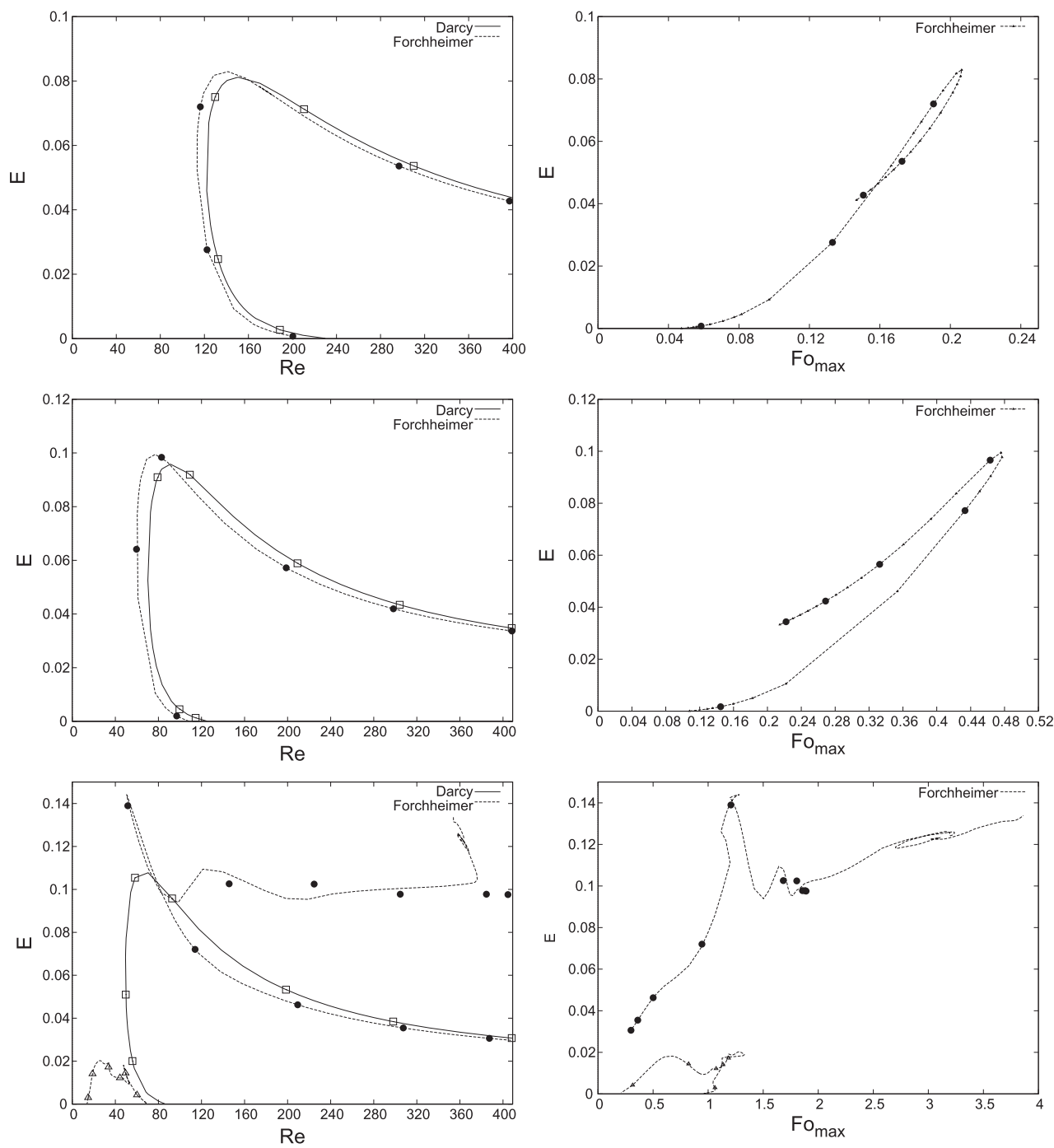

Figure 3. The energy $E$ of the TS waves mapped out in $R e$ (left column) and $F o_{\max }$ (right column, only for the Forchheimer model) for $a=0.40,0.60$ and 0.80 and a streamwise wavenumber $\alpha=0.154$. The legends point out the permeability models. The solutions of the Darcy model correspond to, for all three values of $a$, a truncation of $(N X, N Y)=(14180)$, solid lines, confirmed with (18 200), $\square$ symbols. Top: $a=0.40$, the Forchheimer model solutions are represented by a truncation of (14 180), dashed lines, confirmed by (18 200), $\bullet$ symbols. Middle: $a=0.60$, the Forchheimer model solutions are represented by a truncation of (14 180), dashed lines, confirmed by (18 200), - symbols. Bottom: $a=0.80$, the Forchheimer model solutions are represented by a truncation of (18 180), dashed lines, and confirmed by (22 200), • symbols. The second curve at lower $E$ on the bottom left, connecting together two bifurcation points at $E=0$, is produced by (22 180), dashed line, and confirmed by (26 200), $\triangle$ symbols. 
studied in Wedin et al (2015). This particular value of $\alpha$ is also of interest since it is close to the streamwise wavenumber for which TWs are found at the minimum Reynolds number (for zero or low permeability, see Wedin et al (2015)); it is thus an appropriate value for studying how the minimum Reynolds number changes as $a$ increases. Looking at equations (6) and (7) it is clear that the second term of the Forchheimer model becomes increasingly important with increasing values of $a$ (i.e. the parameter $\hat{K}_{1}$ ) or for sufficiently low values of the Reynolds number (i.e. larger amplitude of the wall suction $\hat{V}_{0}$ ). Hence for these two cases a deviation from the Darcy model is expected, as studied below.

Using the solution characterized by $(\alpha, a)=(0.154,0)$, as an initial guess one can trace out the solutions in $a$ and then study the parameter space ( $\alpha$ and $R e$ ) for each individual value of $a$. For fairly low values of $a$, such as $a=0.01$ and 0.02 the two models yield identical solutions (not presented here). Tracing out the energy $E$ of the TS waves in $R e$ and increasing the permeability to $a=0.40,0.60$ and 0.80 one finds that for the first two values of $a$ a single family of solutions exists, presenting similar, although not identical values of $E$ for a given $R e$ (see left column of figure 3 ). This indicates the relative viability of the Darcy model thus far, although it provides a difference of 8 to $19 \%$ in the determination of the value of the node point $R e_{g}$ (where the solutions bend back) with respect to the Forchheimer model. A strong difference in the bifurcation diagram is first seen for $a=0.80$ where the Forchheimer model gives rise to two families of nonlinear solutions, as shown in the left bottom frame of figure 3 . One solution branch has a bifurcation point (i.e. energy $E=0$ ) at $R e \approx 69$, close to the one of the Darcy model at $R e \approx 86$, and connects with a bifurcation point at a lower Reynolds number at $R e \approx 14$. The other family of solutions found using the Forchheimer model has a higher energy content and does not connect with a bifurcation point in the considered range of Reynolds numbers. As shown in figure 3 the upper branch of this particular curve for $a=0.80$ is more difficult to resolve, as indicated by the slight difference between the two truncations used (compare the dashed lines with the black filled circles in the figure). This branch is shown only as far as convergence can be confirmed as opposed to the lower (less nonlinear) branch that can easily be resolved using various truncations. The lower branch of the higher amplitude family of solutions found for the Forchheimer model is close to the upper branch of the Darcy solutions down to the node, i.e. the point where the branches take two opposite directions in $E$. The figure suggests that for a certain value of $a$ the single family of solutions for $a=0.40$ and 0.60 (referring to both models), splits up in two as can be seen for $a=0.80$ for the Forchheimer model. As discussed in the previous section, the For-chheimer number defined in equation (9) provides an estimate of the magnitude of the difference between the Darcy and the Forchheimer model. Its variation with respect to the energy $E$, for the values of $a$ considered above, is provided in the right column of figure 3 showing the values of $E$ of the solution branches versus $F o_{\max }$, in the range corresponding to the interval in $R e$ shown in the left column. For $a=0.40$ (top frame), along the solution

branches we find values of $F o_{\max } \lesssim 0.2$, rising up to 0.48 for $a=0.60$ (middle frame). For $a=$ 0.80 , at which strong discrepancies are found between the outcomes of the two models,

values of the Forchheimer number of $0.3 \lesssim \not \mathcal{B}_{\text {max }}$ re recovered, suggesting that for $F O_{\max }$ $\gtrsim 0.5$ the inertial effects become non-negligible and the Forchheimer model must be used to provide a complete modeling of the dynamics of wall-normal perturbations on the porous wall. Whereas, as anticipated in the discussion of equation (10), for $F o_{\max } \ll 1$ the inertial effects are negligible with respect to the viscous ones, and the two permeability models should render the same results; this goes along with what is seen in the top and the middle subfigures of figure 3 for $a=0.40$ and 0.60 . When $F o_{\max } \gg 1$, see equation (11), inertial effects are dominating, hence the Forchheimer equation serves as the complete model and as a result strong differences should be observed when comparing the Darcy and the 


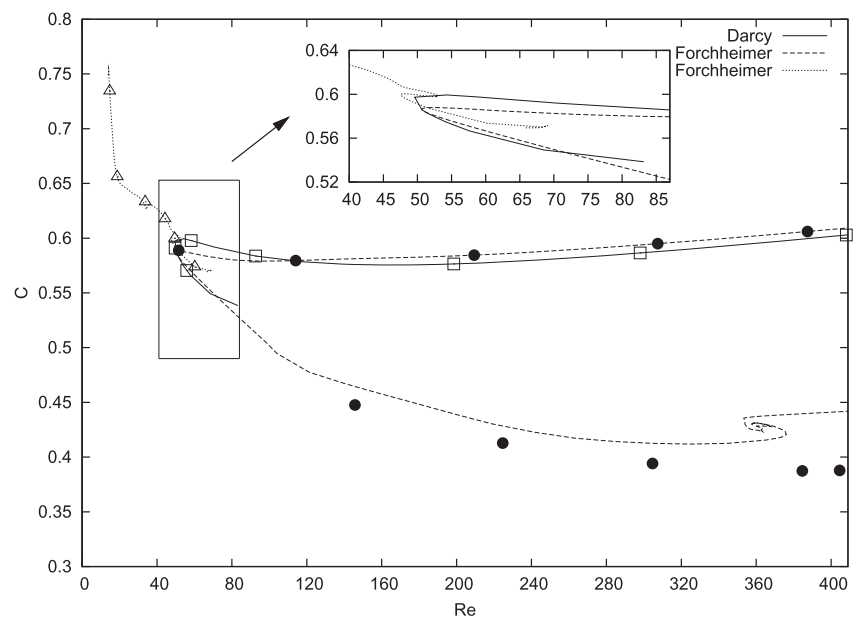

Figure 4. The wave speed $c_{r}$ of the TS waves mapped out in $R e$ for $a=0.80$ and the streamwise wavenumber $\alpha=0.154$. The legends point out the permeability models. The truncations used correspond to those in figure 3. The bifurcation curve of the family of solutions that exist for the Forchheimer model at low Re shown in figure 3 is shown by the dotted line connected by the bifurcation point at $R e \approx 14$ and $R e \approx 69$.

Forchheimer model; this is seen in the bottom subfigure for $a=0.80$. In this particular case, strong discrepancies are already found between the solution branches for $F o_{\max } \gtrsim 0.5$, indicating that even when viscous and inertial effects are comparable in magnitude, considering both effects in the wall-porosity model is crucial to obtain accurate perturbation dynamics. Figure 4 provides additional details on the solution branches found using both permeability models for $a=0.80$ showing the phase speed $c_{r}$ associated with the different families of solutions of figure 3 (bottom left). The narrow family of solutions at low $E$ and $R e$ (i.e. $14 \lesssim R e \lesssim 69$ ) corresponds to an increasing value of $c_{r}$ as one moves to a lower Reynolds number, whereas the high-energy branch found at a larger $R e$ has lower values of the phase speed ranging from approximately $c_{r}=0.40$ to 0.60 .

The previous results have been obtained for a fixed value of $\alpha$; to generalize the discussion, we map out the solutions in $\alpha$ for three fixed values of the permeability $a$ ( $a=0.40$, 0.60 and 0.70 , the latter value being close to the threshold at which strong discrepancies begin to be found between the two different models), at selected values of the Reynolds number $\operatorname{Re}(a)$ (see figure 5). Based on figure 3 the Reynolds number value $\operatorname{Re}(a)$ chosen is the one encompassed by both models and situated fairly close to the nodes, the point where the nonlinear solutions bend back. Moreover, we choose low values of $R e$ in order to consider a case in which it is more likely to find a difference between the Forchheimer and the Darcy model, as can be anticipated by comparing equation (7) for the Forchheimer model to equation (6) for the Darcy one. Thus, for tracing out the solutions in $\alpha$ we select $\operatorname{Re}(a=0.40)=130$, $\operatorname{Re}(a=0.60)=75$ and $\operatorname{Re}(a=0.70)=68$. The solution branches for these values of $a$ and $R e$ are shown in figure 5. For $a=0.40$ and $a=0.60$ (top and middle frames on the left) the Forchheimer and Darcy's branches are rather similar in shape, although not identical. Whereas, for $a=0.70$ there is a tangible deviation of the Forchheimer model away from the Darcy description, with the emergence of a second family of solutions ranging from very low values of $\alpha$ to around 1.5 (the full range in $\alpha$ is not shown). The right column of figure 5 provides the Forchheimer number $F o_{\max }$ associated to the solution branches 

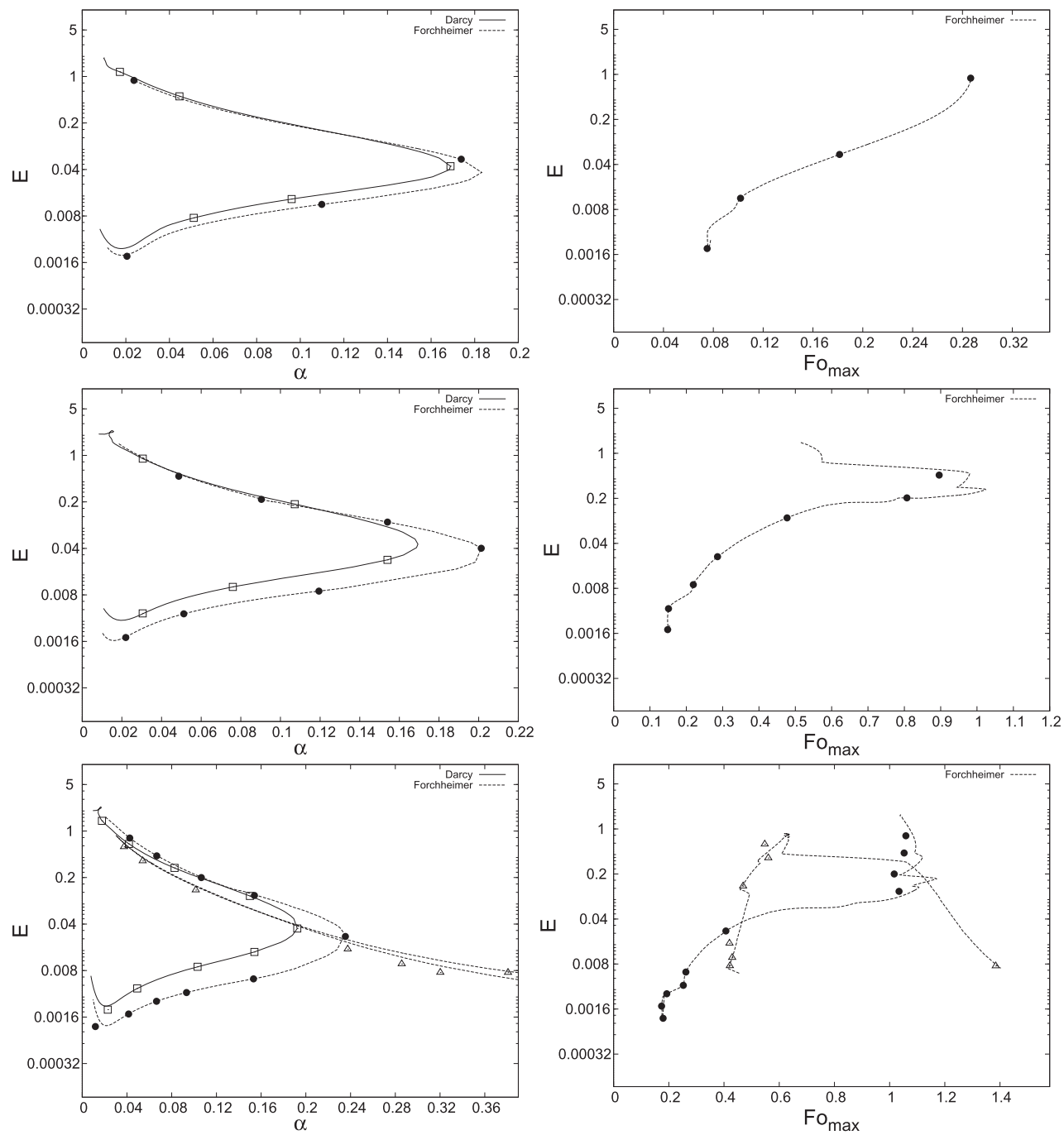

Figure 5. The energy $E$ of the nonlinear solutions mapped out in $\alpha$ (left column) and $F o_{\text {max }}$ (right column, only for the Forchheimer model), for the permeabilities $a=0.40$, 0.60 and 0.70 . The energy $E$ is shown in logscale in order to differentiate the curves. The legends point out the solutions of the Darcy and the Forchheimer model. The solutions of the Darcy model correspond to, for all three values of $a$, a truncation of $(N X, N Y)=(14180)$ and are confirmed with $(18200), \square$ symbols. Top: $a=0.40$ and $R e=130$, the Forchheimer model solutions are represented by a truncation of (14 180) and confirmed by (18 200), symbols. Middle: $a=0.60$ and $R e=75$, the solutions of the Forchheimer model are represented by a truncation of (18 180) and confirmed by (22 200), symbols. Bottom: $a=0.70$ and $R e=68$, the solutions of the Forchheimer model are represented by a truncation of $(14$ 180) and confirmed by $(18200)$, • symbols. The second branch of the Forchheimer model stretching across the shown range of $\alpha$ is represented by (36 160) and confirmed by (40 180), $\triangle$ symbols. In order to have a continuous and well-resolved solution curve the second branch needed a truncation of $N X=36$ terms in $x$. 


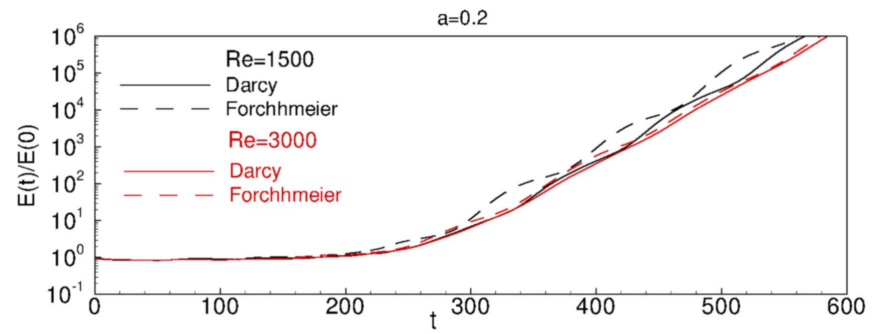

Figure 6. Energy $E$ versus time extracted from a linearized DNS initialized with the TWs computed for $a=0.2, \alpha=0.2, R e=1500$ (black lines) and $R e=3000$ (red lines) for the Darcy (solid lines) and the Forchheimer (dashed lines) permeability models.

shown on the left; confirming the previously-found results, the outcomes of the two permeability models begin to deviate as the Forchheimer number $F o_{\max }$ surpasses a value of about 0.5 . According to both figures 3 and 5 an increasing permeability $a$ is associated with a displacement of the interval of $F o_{\max }$ to higher values. If the value of $a$ (and thus $F o_{\max }$ ) is sufficiently large the Forchheimer model gives rise to a family of solutions not found when using the Darcy model, as shown for $a=0.70$ (figure 5) and 0.80 (figure 3). An important point concerning the mapping out of the solutions in $\alpha$, is that the required value of $y_{\infty}$ increases rather quickly as $\alpha$ reduces below a threshold value, close to 0.05 . This is probably the reason for the difficulty of closing the curves in the range where $\alpha$ is of $O\left(10^{-3}\right)$ to $O\left(10^{-2}\right)$, where the required value of $y_{\infty}$ might be larger than 500. For these low values of $\alpha$ no convergence can be proved. This issue is probably not merely related to the requirement of large values of $y_{\infty}$ but also to the fact that nearly $X$-independent solutions (i.e. $\alpha \approx 0$ ) are represented by a Fourier series being dependent on $X$. A related issue is seen as one increases $\alpha$. The extreme case is the curve stretching across the shown range of $\alpha$ for $a=0.70$ and $\operatorname{Re}=68$ (figure 5, below), where the required $y_{\infty}$ is below 10 at $\alpha \approx 1.5$ (not part of the interval shown in figure 5) and may be the cause of issues in correctly adapting the value of $y_{\infty}$, yielding difficulties in continuing the curve at this other extreme of the spectrum of $\alpha$.

To conclude the discussion, we observe that increasing $a$ makes the computation of nonlinear TS waves possible at much lower Re than those considered in Wedin et al (2015). This implies that alternative solutions start cohabiting the phase space with the laminar flow at ever lower values of the Reynolds number as the wall permeability is increased. Keeping the streamwise wavenumber $\alpha$ fixed to 0.154 , the minimum [Re; $a, \alpha=0.154]$ is brought from around 3000 for zero permeability $a=0$, as found in Wedin et al (2015), down to below 50 for $a=0.80$ for both permeability models studied here. For the Forchheimer model at this particular value of $a$, we find a global minimum $R e \approx 44$ for $\alpha=0.154$. Both the Darcy and the Forchheimer models lead to similar values of the above-mentioned minimum Reynolds number, although the associated bifurcation diagrams deviate notably from each other at low Reynolds numbers and high $a$. A parameter study in $\alpha$ at a low Reynolds number $(R e=68)$ narrows the value at which this strong deviation begins to be found down to $a=0.70 \pm 0.10$ (in dimensional terms $\hat{K}_{1}=6.72^{-9} \times 10 \quad \times \boxminus 196^{9} \mathrm{~m} \quad{ }^{2}$ ). This suggests a value of $a$ for which the Darcy model loses its viability. Moreover, for larger values of $a$, the additional branch of solutions first appearing for $a \geqslant 0.70$ and stretching between $R e \approx 14$ to $R e=50-80$ (depending on the value of $a$ ) disappears. In particular, at $a \approx 1.40$ no bifurcation point at $E=0$ exists in the interval of $R e$ mentioned above. 


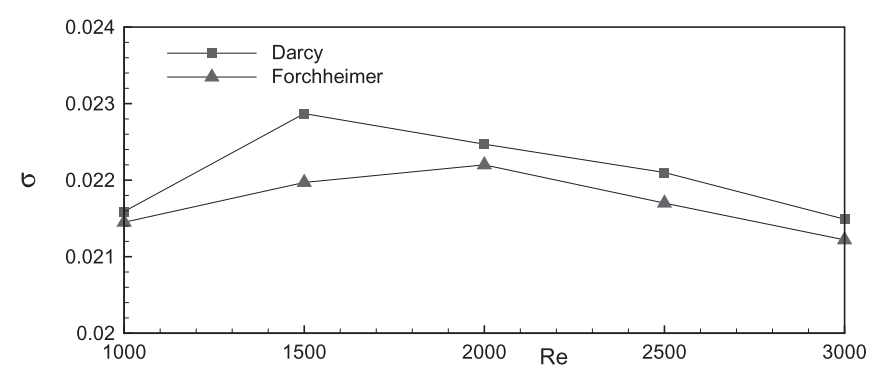

Figure 7. Growth rate $\sigma$ of the unstable mode versus the Reynolds number for the TWs computed with $a=0.2$ and $\alpha=0.2$, for the Darcy (squares) and the Forchheimer (triangles) permeability models.
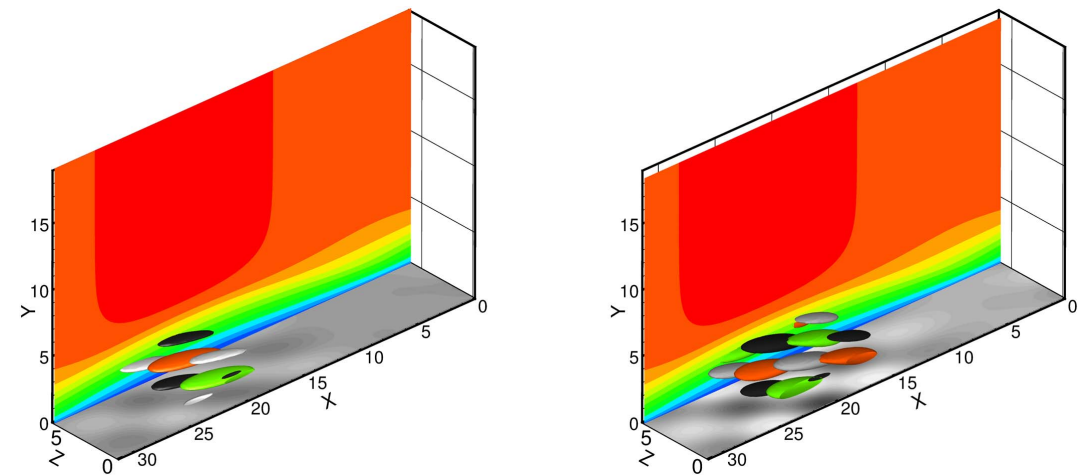

Figure 8. Colored isocontours of the TW streamwise velocity on the plane $z=2 \pi$ and isosurfaces of the streamwise (red and green) and the spanwise (black and white) perturbation to the TW extracted at $t=500$ from a linearized DNS initialized by the upper-branch solution with $\alpha=0.20, R e=1500, a=0.2$ for the Darcy (left) and the Forchheimer model (right). The gray isocontours at the wall represent the wall-suction perturbation.

In this section we have discussed the influence of the Darcy and Forchheimer wallporosity models on the TW solution branches, as the quantities $a, \alpha$ and $R e$ are varied in the parameter space. In the following we study the linear stability of the TS waves, by superposing a perturbation on the considered nonlinear TS waves. In particular, we will focus on fairly low permeability values, for which the obtained TW solutions show slight differences, in order to assess if these mild discrepancies can affect the instability features of the obtained solutions.

\subsection{Stability of the TS waves}

The TW computed at different Reynolds numbers for $a=0.2$ and $\alpha=0.2$ is considered for the two different permeability models, in order to compare their linear stability. For both permeability models, the TWs are found to be unstable with respect to a low-frequency mode, which oscillates with a period of $\approx 100$ while growing in time. Figure 6 shows the integral perturbation energy gain for the two models, at two different Reynolds numbers, $R e=1500$ and $R e=3000$. Although the overall dynamics are similar, with the energy gain remaining close to 1 for a long time before the developing of the unstable mode, the stability of the TW 
is found to change with the Reynolds number. In fact, while for $R e=3000$ the two considered permeability models provide a very similar outcome, for $R e=1500$ the two energy gain curves show non-negligible discrepancies. This indicates that, even if the amplitude and shape of the TW is well approximated by the Darcy model for $a=0.2$, its stability features can be affected by the use of this permeability model instead of the, more accurate, For-chheimer one. This is confirmed by looking at figure 7, where the growth rate $\sigma$ of the unstable modes is evaluated for different Reynolds numbers. For both models the growth rate curve shows a peak followed by an asymptotic decrease of the growth rate, as is also observed by Wang et al (2007) for the Couette flow. However, the peak is found at higher values of the Reynolds number for the Forchheimer model $(R e \approx 2000)$, and, even if the asymptotic scaling appears to be well-described by the Darcy model, the predicted growth rates are up to 5\% larger than the ones found by using the Forchheimer model, for all considered values of $R e$. Some differences are found also in the shape of the unstable mode for the two models. Figure 8 provides the most unstable mode extracted by the DNS at $t=500$ (isosurfaces), together with the associated TWs for $a=0.2, \alpha=0.2$, and $R e=1500$. The TW super-posed to the base flow solution, shown by the colored isocontours in the $z=2 \pi$ plane, induces a streamwise modulation in the thickness of the ASBL flow; comparing the left and the right frame of the figure, one can see that the TWs computed using the two permeability models are almost indistinguishable. The unstable mode is shown in the same figure by the isosurfaces of streamwise (red for positive and green for negative) and spanwise (white for positive and black for negative) perturbation; in both cases it is localized in the streamwise region in which the TW induces a deceleration of the flow, and is composed of different patches of spanwisealternated positive and negative perturbations, inducing a three-dimen-sionalization of the flow structures leading the flow to transition. The alternated pattern of positive and negative streamwise and spanwise perturbations appears to be very similar for both of the considered models. However, for the Forchheimer model the mode is more extended in the streamwise direction, presenting alternating perturbation patches also in the streamwise direction. This will allow a more rapid streamwise spreading of the mode during the transition to turbulence. These discrepancies between the two models will be certainly more pronounced for larger permeabilities; however, we have not been able to verify this point since the DNS code suffered from instability issues for large values of the permeability. However, the results presented for $a=0.2$ already prove that the instability features of the TW are not perfectly described by the Darcy model.

\section{Conclusions}

The nonlinear stability of a boundary-layer flow over a permeable wall is considered, with the aim of comparing two models of permeability, the Darcy and the Forchheimer description, to gain insight into the viability of the supposedly less-accurate Darcy model. To this end, nonlinear TS waves are mapped out in parameter space, and their three-dimensional linear stability is studied, using both permeability models, to compare the obtained results. For low values of the non-dimensional wall permeability $a$ the solutions provided by the two models are similar in amplitude and shape, and the bifurcation branches are sufficiently close in the parameter space. Increasing the wall permeability to values $a \gtrsim 0.7$ (in dimensional terms being $\hat{K}_{1} \gtrsim 6.72 \cdot 10^{-9} \mathrm{~m}^{2}$ ) leads to strong differences in the bifurcation branches, with the emergence of a new solution branch for $R e<100$. The associated Forchheimer number, $F O_{\text {max }}$, providing the ratio between kinetic and viscous forces at the porous wall, changes from $F o_{\max } \lesssim 0.5$ for $a=0.60$ to $F o_{\max } \gtrsim 0.5$ for $a=0.80$, implying that above this value 
both viscous and inertial effects at the wall become important and cannot be neglected. Moreover, when increasing $a$ from zero the solution branches move towards lower values of $R e$. Keeping the streamwise wavenumber $\alpha$ fixed to 0.154 (value used in Wedin et al (2015)) the global minimum of the Forchheimer model is found to be situated at $R e \approx 44$ for $a=0.80$. The previous study by Wedin et al (2015) has shown that, increasing the wall permeability using the Darcy model, the solution branches strongly departs from the ones found imposing a no-slip condition. The results contained herein deepen the previous analysis indicating that, for high permeabilities, the Forchheimer condition at the plate should be considered instead of the simplified Darcy model. This is confirmed by an instability study performed using a linearized DNS in a three-dimensional framework, showing that, already for $a=0.2$, some differences are found in the asymptotic stability of the solutions obtained by using the two different models. In particular, the Darcy model appears to overestimate the growth rate of the unstable mode at all Reynolds numbers, and fails to predict the Reynolds number value at which the growth rate peaks. The overall shape of the unstable mode appears well predicted, except for its streamwise extension, which is underestimated by the Darcy model. The results contained herein indicate that for studying the transition to turbulence of the flow over a permeable wall the underlying permeability model must be carefully chosen to correctly reproduce the kinetic and viscous forces at the wall, and their influence on the perturbation dynamics.

\section{Acknowledgments}

The financial support of the Marie Curie European Reintegration Grant (ERG) No. 268265 is gratefully acknowledged by H.W. The authors would like to thank A. Bottaro, University of Genova, for the fruitful discussions which inspired the main idea of the present paper and also M. D. de Mello Innocentini, University of Ribeirão Preto, for valuable help with the Forchheimer model.

\section{References}

Acarlar M and Smith C 1987a J. Fluid Mech. 175 1-41

Acarlar M and Smith C 1987b J. Fluid Mech. 175 43-83

Brand E and Gibson J 2014 J. Fluid Mech. 750 R3

Chandler G and Kerswell R 2013 J. Fluid Mech. 722 554-95

Chantry M, Willis A and Kerswell R 2014 Phys. Rev. Lett. 112164501

Cherubini S, Palma P D and Robinet J C 2015 Phys. Fluids 27034108

Cvitanović P 2013 J. Fluid Mech. 726 1-4

Cvitanović P, Artuso R, Mainieri R, Tanner G and Vattay G 2016 Chaos: Classical and Quantum (Niels Bohr Institute, Copenhagen) (http://chaosbook.org/)

Davidsson E 2007 Stability and transition in the suction boundary layer and other shear flows $P h D$ Thesis Luleå University of Technology, Sweden

Deguchi K and Hall P 2014 J. Fluid Mech. 752 602-25

Emmons H W 1951 J. Aero. Sci. 18 490-8

Emmons H W and Bryson A E 1952 Proc. First U.S. Nat. Cong. Appl. Mech. (June 1951, Chicago, 111.), A.S.M.E 859-68

Faisst H and Eckhardt B 2003 Phys. Rev. Lett. 91224502

Forchheimer P 1901 Zeitschr. Ver. Dtsch. Ing 45 1736-41

Fransson J 2003 Flow control of boundary layers and wakes PhD Thesis Royal Institute of Technology, KTH Mechanics

Fransson J and Alfredsson P 2003 J. Fluid Mech. 482 51-90

Fransson J and Corbett P 2003 Eur. J. Mech. B/Fluids 22 259-70 
Gibson J and Brand E 2014 J. Fluid Mech. 745 25-61

Gustavsson C 2000:017 Master Thesis Luleå University of Technology, Sweden (available at: http:// pure.ltu.se/portal/en/studentthesis/search.html?search=Development + of + threedimensional + disturbances + in + boundary+layers + with + suction\&uri $=$ )

Hagen G H L 1839 Poggendorf's Annalen der Physik und Chemie 46 423-42

Hocking L 1975 Quart. J. Mech. Appl. Math. 28 341-53

Innocentini M, Salvini V, Coury J and Pandofelli V 1999 Am. Ceram. Soc. Bull. 82 78-84

Innocentini M, Sepulveda P and Ortega F 2005 Cellular Ceramics. Structure, Manufacturing, Properties and Applications ed M Scheffler and P Colombo (Weinheim, Germany: Wiley-VCH) pp 313-41

Innocentini M, Tanabe E, Aguiar M and Coury J 2012 Chem. Eng. Sci. 74 38-48

Itano T and Generalis S 2009 Phys. Rev. Lett. 102114501

Kawahara G and Kida S 2001 J. Fluid Mech. 449 291-300

Khapko T, Kreilos T, Schlatter P, Duguet Y, Eckhardt B and Henningson D 2013 J. Fluid Mech. 717 R6

Kline S, Reynolds W, Schraub F and Runstadler P 1967 J. Fluid Mech. 30 741-73

Kreilos T, Veble G, Schneider T and Eckhardt B 2013 J. Fluid Mech. 726 100-22

Lee M K, Eckelman L D and Hanratty T J 1974 J. Fluid Mech. 66 17-33

Levin O, Davidsson E and Henningson D 2005 Phys. Fluids 17114104

Lucas D and Kerswell R 2015 Phys. Fluids 27045106

Milinazzo F and Saffman P 1985 J. Fluid Mech. 160 281-95

Nagata M 1990 J. Fluid Mech. 217 519-27

Pluvinage F, Kourta A and Bottaro A 2015 Phys. Fluids. 27054104

Poiseuille J 1846 C. R. Acad. Sci. 11 961-7

Pringle C, Duguet Y and Kerswell R 2009 Phil. Trans. Roy. Soc. A 367 457-72

Rayleigh J 1892 Phil. Mag. 34 59-70

Reynolds O 1883 Phil. Trans. 174 935-82

Reynolds O 1895 Phil Trans. R. Lond. Soc. A 186 123-64

Reynolds O 1900 Papers on Mechanical and Physical Subjects (Cambridge: Cambridge University Press)

Rheinboldt W and Burkardt J V 1983a ACM Trans. Math. Softw. 9 236-41

Rheinboldt W and Burkardt J V 1983b ACM Trans. Math. Softw. 9 215-35

Ruth D and Ma H 1992 Transp. Porous Media 7 255-64

Schlichting H 1933 Nachr. Ges. Wiss. Göttingen Math. Phys. Klasse 181-208

Schmid P and Henningson D 2001 Stability and Transition in Shear Flows (Berlin: Springer)

Tollmien W 1929 Nachr. Ges. Wiss. Göttingen, Math. Phys. Klasse 21-44

Uhlmann M, Kawahara G and Pinelli A 2010 Phys. Fluids 22084102

Verzicco R and Orlandi P 1996 J. Comp. Phys. 123 402-14

Waleffe F 1998 Phys. Rev. Lett. 81 4140-3

Waleffe F 2001 J. Fluid Mech. 435 93-102

Waleffe F 2003 Phys. Fluids 15 1517-34

Wang J, Gibson J and Waleffe F 2007 Phys. Rev. Lett. 98204501

Wedin H, Bottaro A, Hanifi A and Zampogna G 2014 Eur. Phys. J. E, Soft Matter and Biological Physics 3734

Wedin H, Bottaro A and Nagata M 2009 Phys. Rev. E 79 065305(R)

Wedin H, Cherubini S and Bottaro A 2015 Phys. Rev. E 92013022

Wedin H and Kerswell R 2004 J. Fluid Mech. 508 333-71 